\title{
Des critères d'assurance désuets empêchent le remboursement de soins fondés sur des données probantes
}

\author{
Ahmed Bayoumi MD MSc MPA, Andreas Laupacis MD MSc
}

Citation : CMAJ 2021 October 12;193:E1573-4. doi : 10.1503/cmaj.211617-f

Voir la version anglaise de l'article ici : www.cmaj.ca/lookup/doi/10.1503/cmaj.211617; voir les articles connexes en anglais ici : www. cmaj.ca/lookup/doi/10.1503/cmaj.210523 et www.cmaj.ca/lookup/doi/10.1503/cmaj.210811

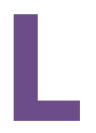

es conclusions de 2 articles sur l'utilité clinique des anticoagulants oraux directs (AOD) publiés dans le $C M A J^{1,2}$ mettent en lumière une importante faille dans les processus décisionnels des régimes publics d'assurance-médicaments au Canada en ce qui concerne le remboursement de ces médicaments. Kimpton et ses collègues ont constaté que l'apixaban était à la fois plus efficace et moins coûteux que les autres options lorsqu'il est administré pour la thromboprophylaxie veineuse primaire chez les patients atteints de cancer ${ }^{1}$; Grewal et ses collègues ont quant à eux montré que les personnes âgées ayant subi un traumatisme crânien qui prenaient des AOD étaient moins susceptibles de présenter une hémorragie intracrânienne que les patients sous warfarine².

Ces conclusions concordent avec un important corpus de recherches selon lesquelles les $A O D$, qui sont approuvés et disponibles depuis plus d'une décennie, sont des médicaments efficaces qui présentent un risque hémorragique généralement moindre que la warfarine ${ }^{3}$. Le National Institute for Health and Care Excellence du Royaume-Uni, qui fonde ses recommandations sur des données probantes concernant le rapport coûtefficacité, préconise les AOD comme anticoagulants de première intention chez les patients qui font de la fibrillation auriculaire ${ }^{4}$. Or, au Canada, les régimes publics d'assurancemédicaments continuent de restreindre la prescription des AOD. En Colombie-Britannique, en Alberta et en Ontario, les listes provinciales de médicaments remboursés ne comprennent pas l'apixaban pour la prévention des AVC chez les patients qui font de la fibrillation auriculaire (l'indication la plus courante de l'anticoagulothérapie orale chez les personnes âgées), à moins qu'ils aient obtenu une anticoagulation inadéquate avec la warfarine ou que cette dernière leur soit contre-indiquée ${ }^{5-7}$.

Pourquoi la pratique tire-t-elle autant de l'arrière par rapport aux données probantes? Malheureusement, au Canada, les décisions qui se prennent peu après l'approbation initiale d'un médicament sont rarement mises à jour quand de nouvelles données deviennent disponibles. Cela fait en sorte que, pour pouvoir offrir le traitement le meilleur et le plus sécuritaire, certains médecins doivent déjouer le système en affirmant que leurs patients répondent aux critères de remboursement quand ce n'est pas le cas. Les médecins déjoueront le système s'ils estiment que l'entorse à la règle ( $p$. ex., déclarer faussement qu'un patient ne tolère pas la warfarine) est dans l'intérêt de leur patient. Le fait que près de 3 fois plus de patients d'une étude ontarienne sur les hémorragies intracrâniennes chez des patients sous anticoagulothérapie prennent un AOD plutôt que de la warfarine ${ }^{2}$ illustre l'ampleur des ruses probablement utilisées par les médecins, car la véritable intolérance à la warfarine est rare. Les éthiciens médicaux défendent le recours à de tels subterfuges dans les cas où les médecins estiment que le bienêtre d'un patient se trouve compromis par un système de santé inéquitable ou dysfontionnel ${ }^{8}$.

À l'instar de nombreux médecins, nous croyons approprié que les régimes publics d'assurance-médicaments ne remboursent pas les médicaments qui ont un mauvais rapport coût-efficacité, car pour le bien public, les ressources en santé doivent être utilisées judicieusement. Par contre, comme les connaissances évoluent, le processus décisionnel concernant le remboursement des médicaments devraient être itératif.

Dans la plupart des régimes publics d'assurance-médicaments canadiens, les décisions relatives au remboursement reposent sur les recommandations du Comité canadien d'expertise sur les médicaments de l'Agence canadienne des médicaments et des technologies de la santé 9 . Le Québec s'est doté d'un processus parallèle mais indépendant. Il y a une forte concordance entre ces recommandations et les décisions finales quant au remboursement. Presque toutes les nouvelles recommandations s'accompagnent de critères pour limiter l'usage, en restreignant la prescription à certains types de patients ou selon l'expertise des médecins. 
Il y a peu de possibilités de modifier ces décisions une fois qu'elles ont été prises. Un fabricant pharmaceutique peut demander d'ajouter des indications pour un médicament si de nouvelles données voient le jour, et un régime d'assurancemédicaments peut demander une révision des critères de remboursement pour un médicament ou une classe de médicaments (comme les anticoagulants). Par contre, l'arrivée de nouvelles données probantes importantes n'entraîne pas systématiquement une telle révision.

Lorsque les critères de remboursement de médicaments deviennent désuets, personne n'y gagne. Les médecins font face à un choix délicat entre défendre les intérêts de leurs patients ou observer strictement les normes professionnelles en vigueur. Les patients en souffrent parce que les médecins qui suivent ces directives gouvernementales désuètes leur offrent des soins sous-optimaux; et les systèmes de santé sont perçus comme inertes face à l'émergence de données de grande qualité lorsqu'ils tolèrent que leurs règles soient contournées.

Trois changements à ces processus pourraient aider à résoudre ce problème. Premièrement, les recommandations devraient s'accompagner d'une date de révision préétablie ou de revues périodiques des données probantes. Les lignes directrices du National Institute for Health and Care Excellence incluent un processus actif de surveillance des nouvelles données publiées et une vérification formelle des données probantes tous les 5 ans $^{10}$.

Deuxièmement, il pourrait être avantageux de permettre à d'autres instances de demander une révision. Tant les sociétés cliniques (p. ex., la Société canadienne d'hématologie) que les groupes de défense des patients (p. ex., la Fondation des maladies du cœur et de l'AVC) pourraient être justifiés de demander une révision des critères différente de celle des fabricants ou des régimes d'assurance publics. Un processus qui permettrait à ces groupes de demander qu'on revoie les critères rendrait le système beaucoup mieux centré sur les patients.

Troisièmement, les régimes publics d'assurance-médicaments pourraient régulièrement analyser les habitudes d'ordonnances pour vérifier dans quelle mesure les restrictions sont contournées et, le cas échéant, déclencher un examen des critères.

La révision périodique des médicaments alourdira la tâche et demandera des ressources additionnelles pour les comités responsables du remboursement des médicaments, mais il est essentiel qu'ils mettent à jour les listes de médicaments régulièrement si l'on veut disposer de régimes publics d'assurancemédicaments fiables, adaptés et efficients.

\section{Références}

1. Kimpton M, Kumar S, Wells PS, et al. Cost-utility analysis of apixaban compared with usual care for primary thromboprophylaxis in ambulatory patients with cancer. CMAJ 2021;193:E1551-60.

2. Grewal K, Atzema CL, Austin PC, et al. Intracranial hemorrhage after head injury among older patients on anticoagulation seen in the emergency department: a population-based cohort study. CMAJ 2021;193:E1561-7.

3. Chen A, Stecker A, Warden BA. Direct oral anticoagulation use: a practical guide to common clinical chellanges. J Am Heart Assoc 2020;9:e017559.

4. Atrial fibrillation: diagnosis and management. London (UK): National Institute for Health and Care Excellence; 2021. Accessible ici : https://www.nice.org.uk/ guidance/ng196 (consulté le 13 sept. 2021).

5. Alberta Drug Benefit List. Edmonton: Alberta Blue Cross/Alberta Health; 2021. Accessible ici : https://www.ab.bluecross.ca/dbl/pdfs/dbl_full_list.pdf (consulté le 13 sept. 2021).

6. Formulary search. Toronto: Government of Ontario; 2021. Accessible ici : https://www.formulary.health.gov.on.ca/formulary/ (consulté le 13 sept. 2021).

7. Limited coverage drugs - apixaban for atrial fibrillation (AF). Vancouver: Province of British Columbia. Accessible ici : https://www2.gov.bc.ca/gov/content/health/ practitioner-professional-resources/pharmacare/prescribers/limited-coverage-drug -program/limited-coverage-drugs-apixaban-for-atrial-fibrillation-af (consulté le 13 sept. 2021).

8. Tavaglione N, Hurst SA. Why physicians ought to lie for their patients. Am J Bioeth 2012;12:4-12.

9. Canadian Drug Expert Committee (CDEC). Ottawa: CADTH; 2017. Accessible ici : https://www.cadth.ca/canadian-drug-expert-committee-cdec (consulté le 13 sept. 2021).

10. Developing NICE guidelines: the manual: ensuring that published guidelines are current and accurate. London (UK): National Institute for Health and Care Excellence; updated 2020 Oct. 15. Accessible ici : https://www.nice.org.uk/process/pmg20/ chapter/ensuring-that-published-guidelines-are-current-and-accurate (consulté le 13 sept. 2021).

Intérêts concurrents : Ahmed Bayoumi est membre du Comité canadien d'expertise sur les médicaments. Son mandat se termine en septembre 2021. Il a reçu des subventions (versées à son établissement) des Instituts de recherche en santé du Canada, de la Fondation de l'Hôpital St. Michael, de la Canadian Research Initiative in Substance Misuse, de Mitacs et de l'Université de Toronto. Voir : www.cmaj.ca/site/misc/cmaj_staff.xhtml pour Andreas Laupacis.

Affiliations : Centre for Urban Health Solutions (Bayoumi), Hôpital St. Michael, Unity Health Toronto, Toronto, Ont.; rédacteur adjoint principal, CMAJ (Laupacis).

Propriété intellectuelle du contenu : Il s'agit d'un article en libre accès distribué conformément aux modalités de la licence Creative Commons Attributions (CC BY-NC-ND 4.0), qui permet l'utilisation, la diffusion et la reproduction dans tout médium à la condition que la publication originale soit adéquatement citée, que l'utilisation se fasse à des fins non commerciales (c.-à-d. recherche ou éducation) et qu'aucune modification ni adaptation n'y soit apportée. Voir : https://creativecommons.org/licenses/by-nc-nd/4.0/deed.fr.

Correspondance : Rédaction du CMAJ, editorial@cmaj.ca 